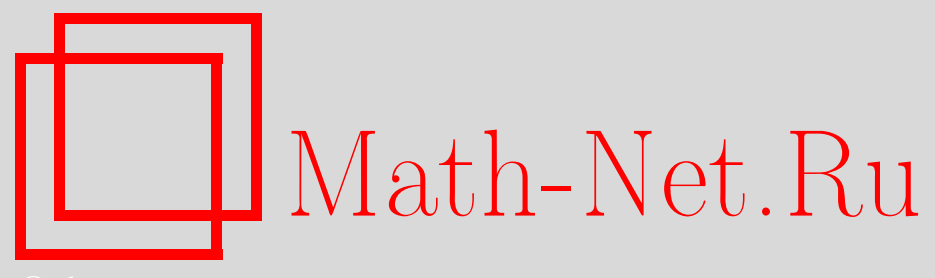

А. Корчевский, О точных степенях и не только, Квант, 2019, номер 6, 2-7

DOI: https://doi.org/10.4213/kvant20190601

Использование Общероссийского математического портала Math-Net.Ru подразумевает, что вы прочитали и согласны с пользовательским соглашением http://www.mathnet.ru/rus/agreement

Параметры загрузки:

IP : 3.80 .253 .173

26 апреля 2023 г., 16:53:13

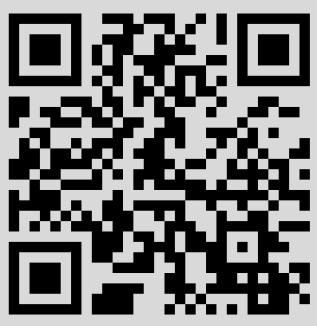




\section{О точных степенях и не только}

\section{А.КОРЧЕВСКИЙ}

ОКРОВИЩНИЦА ТЕОРИИ ЧИСЕЛ, ИЛИ

арифметики, как эту область называли раньше, поистине неисчерпаема. Многие тайны простых чисел сегодня раскрыты. Но перенесем луч света на другие замечательные числовые последовательности - и загадок у них может быть отнюдь не меньше, чем у старых добрых и таких непростых «простых» чисел.

В начале 80-х годов прошлого века, будучи учеником физико-математической школы, автор интересовался различными «расширениями» понятия простых чисел. Простые числа (как наши читатели, безусловно, помнят) - это те самые, которые не делятся ни на одно число, кроме 1 и самого себя. Из простых чисел, как из кирпичиков, складываются все остальных числа, и скрепляющий цемент - операция умножения. Что если мы попробуем «склеивать» ряд натуральных чисел с помощью другого раствора - например, операции возведения в степень? Так автором была придумана «игра в целые степени», которой были посвящены долгие часы досуга. Пользуясь образцом простых чисел, можно попытаться построить целый город или даже королевство, где жили бы их родственники - натуральные числа, которые нельзя представить в виде степени $a^{b}$, где $a$ и $b-$ натуральные числа, $b>1$. Приглашаем читателя поучаствовать в этой игре. Возможно, кто-то сможет выдумать и другие игры, похожие на эту. Милости просим, а пока...

\section{Королевство точных степеней}

Выпишем несколько первых членов последовательности точных степеней:

$$
1,4,8,9,16,25,27,36, \ldots
$$

DOI: https://doi.org/10.4213/kvant20190601
Если же натуральное число не является точной степенью, будем для краткости называть его HEстепенью. (По-английски точные степени именуются perfect powers, а числа, не являющиеся точными степенями, - not perfect powers или, более коротко, non-powers. Будучи школьником, автор придумал для НЕстепеней имя sonor, или «сонорные числа», так что наше королевство можно называть «королевством сонорных чисел».)

Итак, НЕстепени - это:

$2,3,5,6,7,10,11,12,13, \ldots$

Не составляет труда показать, что существует бесконечное количество НЕстепеней (например, поскольку каждое простое число является НЕстепенью).

Упражнение 1. а) Докажите, что если для некоторого простого числа $p$ число $n$ делится на $p$, но не делится на $p^{2}$, то оно не является точной степенью. б) Докажите, что множество составных НЕстепеней бесконечно.

В каталоге целочисленных последовательностей oeis.org последовательность точных степеней имеет код А001597, а НЕстепени - код А007916. В этом же каталоге можно найти ссылки на интересные статьи о свойствах последовательностей.

\section{Основная теорема}

Основная теорема арифметики говорит о том, что каждое натуральное число $N>1$ может быть единственным образом (с точностью до порядка сомножителей) представлено в виде $N=p_{1}^{\alpha_{1}} p_{2}^{\alpha_{2}} \ldots p_{n}^{\alpha_{n}}$, где $p_{1}, p_{2}, \ldots, p_{n}$ являются различными простыми числами, а $\alpha_{1}, \ldots, \alpha_{n}-$ натуральные числа.

Аналог этой теоремы есть и для НЕстепеней. Если число $N=p_{1}^{\alpha_{1}} p_{2}^{\alpha_{2}} \ldots p_{n}^{\alpha_{n}}$ является точной $t$-й степенью, то $t$ должно быть делителем каждого из значений $\alpha_{1}, \ldots, \alpha_{n}$. 
Тогда понятно, что НОД $\left(\alpha_{1}, \ldots, \alpha_{n}\right)=1$, если $N-$ НЕстепень и НОД $\left(\alpha_{1}, \ldots, \alpha_{n}\right)>1$, если $N$ - точная степень. Теперь мы можем определить «наибольший целый корень» для каждого натурального $N$ как наибольшее натуральное $t$, при котором $N^{1 / t}$ является целым. Ясно, что $t=$ НОД $\left(\alpha_{1}, \ldots, \alpha_{n}\right)$. Из этого утверждения сразу следует, что наибольший целый корень $t$ существует для любого $N>1$ и что при этом $N^{1 / t}-$ НЕстепень. Отсюда, мы можем вывести следующий факт.

Теорема 1. Каждое натуральное $N>1$ может быть представлено единственным образом в форме $N=m^{t}$, где $m-$ HЕстепень, а $t$ - натуральное.

Упражнение 2. Докажите, что если $N$ одновременно равно некоторой степени натурального числа $a$ и некоторой степени натурального $b$, то $a$ и $b$ являются степенями некоторого натурального $c$.

Как следствие теоремы 1, можем получить следующий любопытный факт.

Каждое натуральное $N \geq 2$ может быть единственным образом представлено в виде башни степеней (так называемая power tower) $N=m_{1}^{m_{2}{ }^{m_{k}}}$, где $k-$ некоторое натуральное число, а $m_{1}, m_{2}, \ldots, m_{k}-$ НЕстепени.

Доказательство. Применим индукцию по $N$. Если $N$ - НЕстепень (в частности, если $N=2$ ), то $k=1$ и $m_{1}=N$, иначе, в согласии с представлением $N=m^{t}, t \geq 2$, из теоремы 1 , имеем $m_{1}=m$, и теперь достаточно для $t<N$ воспользоваться существованием и единственностью представления $t=m_{1}^{m_{2}{ }^{m_{k}}}$.

\section{"Степенное решето"}

Для нахождения всех простых чисел не больше заданного числа имеется известный несложный алгоритм, называемый решетом Эратосфена. Суть этой процедуры такова: среди чисел $2,3, \ldots, N$ берем наименьшее простое число (это число 2) и вычеркиваем все числа, кратные ему, кроме него самого. На следующем этапе берем наименьшее невычеркнутое число и снова вычеркиваем все числа, кратные ему, кроме него самого. Действуем так далее. В результате вычеркнутыми (хотя бы один раз) окажутся все составные числа, а простые останутся невычеркнутыми.

Отталкиваясь от нашей основной теоремы 1, мы может построить аналог решета для НЕстепеней. Эта схема проиллюстрирована на рисунке 1: берем наименьшую

\begin{tabular}{|c|c|c|c|c|c|c|c|c|}
\hline$m$-НЕстепень & 2 & 3 & 5 & 6 & 7 & 10 & $*$ & $*$ \\
\hline$m^{2}$ & 4 & 9 & 25 & 36 & 49 & 100 & $*$ & $*$ \\
\hline$m^{3}$ & 8 & 27 & 125 & 216 & 343 & 1000 & $*$ & $*$ \\
\hline$*$ & $*$ & $*$ & $*$ & $*$ & $*$ & $*$ & $*$ & $*$ \\
\hline$*$ & $*$ & $*$ & $*$ & $*$ & $*$ & $*$ & $*$ & $*$ \\
\hline$m^{k}$ & $2^{k}$ & $3^{k}$ & $5^{k}$ & $6^{k}$ & $7^{k}$ & $10^{k}$ & $*$ & $*$ \\
\hline$*$ & $*$ & $*$ & $*$ & $*$ & $*$ & $*$ & $*$ & $*$ \\
\hline
\end{tabular}

Рис. 1. "Степенное решето»

НЕстепень (это число 2) и вычеркиваем все его степени (первый столбец), кроме него самого. Находим наименьшее невычеркнутов число, оно будет НЕстепенью, снова вычеркиваем его степени и т.д.

Каждое натуральное число $N>1$ может быть найдено в данной таблице один и только один раз.

\section{«На границах королевства»}

Поговорим о распределении точных степеней «ближе к бесконечности».

Читателям, которые интересовались проблемами простых чисел, наверняка известны результаты о функции $\pi(x)$, равной количеству простых чисел, не превышающих $x: \lim _{x \rightarrow+\infty} \frac{\pi(x)}{x}=0$ и более точная асимптотика $\lim _{x \rightarrow+\infty} \frac{\pi(x) \ln x}{x}=1$.

Картина «степенного решета» может создать впечатление, что НЕстепени встречаются в натуральном ряду реже точных степеней: в таблице на рисунке 1 НЕстепени занимают только одну горизонтальную строку, по сравнению с бесконечным количеством рядов, занимаемых точными степенями. В действительности это не так. 
НЕстепени составляют доминирующую часть натурального ряда (рис.2). Среди первых 100 миллионов натуральных чисел 99,98\% являются НЕстепенями, т.е.

\begin{tabular}{|l|l|l|}
\hline \multicolumn{1}{|c|}{$x$} & \multicolumn{1}{|c|}{$p(x)$} & $\begin{array}{l}\text { Доля } p(x) \text { от } x \\
\text { (в процентах) }\end{array}$ \\
\hline 10 & 4 & 40 \\
\hline 100 & 13 & 13 \\
\hline 1000 & 41 & 4,1 \\
\hline 10000 & 125 & 1,25 \\
\hline 100000 & 367 & 0,367 \\
\hline 1000000 & 1111 & 0,1111 \\
\hline 10000000 & 3395 & 0,03395 \\
\hline 100000000 & 10491 & 0,010491 \\
\hline 1000000000 & 32670 & 0,003267 \\
\hline 10000000000 & 102231 & 0,00102231 \\
\hline 100000000000 & 320990 & 0,00032099 \\
\hline 1000000000000 & 1010196 & 0,00010102 \\
\hline 10000000000000 & 3184139 & 0,00003184 \\
\hline 100000000000000 & 10046921 & 0,00001005 \\
\hline 1000000000000000 & 31723592 & 0,00000317 \\
\hline
\end{tabular}

Рис. 2. Количество $p(x)$ точных степеней, не превышающих $x$

точная степень - довольно редкая находка в натуральном ряду, а НЕстепеней, наоборот, «очень много». Далее, чтобы сформулировать точные результаты, введем функцию $p(x)$ - количество точных степеней, не превышающих положительного числа $x$.

Теорема 2. a) $\lim _{x \rightarrow+\infty} \frac{p(x)}{x}=0$.

б) $\lim _{x \rightarrow+\infty} \frac{p(x)}{\sqrt{x}}=1$.

Доказательство. а) Для доказательства этого пункта достаточно провести грубую оценку количества точных степеней $a^{b}$, не превосходящих некоторого $x>1$. При фиксированном $b \geq 2$ имеем $a \leq \sqrt[b]{x} \leq \sqrt{x}$, так как при $a>\sqrt[b]{x}$ получим $a^{b}>x$. Можно считать, что $b \leq \log _{2} x$, так как при $a \geq 2$ и $b>\log _{2} x$ число $a^{b}$ будет больше $x$ (а случай $a=1$ приводит к $a^{b}=1$, которое уже учтено при $b=2$ ). Таким образом, всего остается в рассмотрении не более $\sqrt{x} \log _{2} x$ пар $a, b$, для которых $a^{b} \leq x . \quad$ Отсюда $\quad p(x) \leq \sqrt{x} \log _{2} x \quad$ и $\frac{p(x)}{x} \leq \frac{\log _{2} x}{\sqrt{x}}$. Из стандартного курса анализа известно, что $\lim _{x \rightarrow+\infty} \frac{\log _{\alpha} x}{x^{\beta}}=0$ для любых $\alpha>0, \beta>0$. Отсюда получаем требуемое.

б) У точним оценку из пункта а). Прежде всего заметим, что имеется $[\sqrt{x}]$ точных квадратов, не превосходящих $x$, в частности $p(x) \geq[\sqrt{x}]$. При фиксированном $b \geq 3$ имеем $a \leq \sqrt[b]{x} \leq \sqrt[3]{x}$ и можем считать, что $b \leq \log _{2} x$. Итого $\sqrt{x}-1 \leq$ $\leq p(x) \leq \sqrt{x}+\sqrt[3]{x} \log _{2} x . \quad$ Поскольку $\lim _{x \rightarrow+\infty} \frac{\sqrt[3]{x} \log _{2} x}{\sqrt{x}}=0$, получаем требуемое.

Теорема доказана.

Среди всех точных степеней чаще всего встречаются точные квадраты. Утверждение б) теоремы 2 согласуется с этим неформальным тезисом.

\section{Близкие точные степени}

Из теоремы 2 можно вывести, что для любого заданного $N$ в натуральном ряду можно отыскать $N$ подряд идущих НЕстепеней. Тем не менее, покажем, как отыскать длинный отрезок натурального ряда, состоящий из НЕстепеней, конструктивно. Для этого можно воспользоваться упражнением 1, а. Достаточно, например, потребовать, чтобы первое число в ряду было четным, но не делилось на 4, второе делилось на 3 , но не на 9, и т.д., $i$-е число в ряду делилось на очередное простое $p$, но не на $p^{2}$.

Упражнение 3. Реализуйте этот план, например, рассматривая систему сравнений $x+i \equiv p_{i}\left(\bmod p_{i}^{2}\right), i=1, \ldots, k$.

Указание. Воспользуйтесь китайской теоремой об остатках.

С вопросом о том, каким образом (и как далеко друг от друга) расположены в 
натуральном ряду точные степени, связаны многие знаменитые теоретико-числовые задачи и гипотезы (в некотором смысле, великая теорема Ферма относится к той же области вопросов).

Можно рассмотреть разности между последовательными точными степенями. Отсчитаем сто таких разностей, начиная с самой первой. Затем переместимся к следующей точной степени и снова подсчитаем среднее между ста разностями. Таким образом строится так называемое скользящее среднее. На рисунке 3 показано, как ведет себя это среднее при возрастании

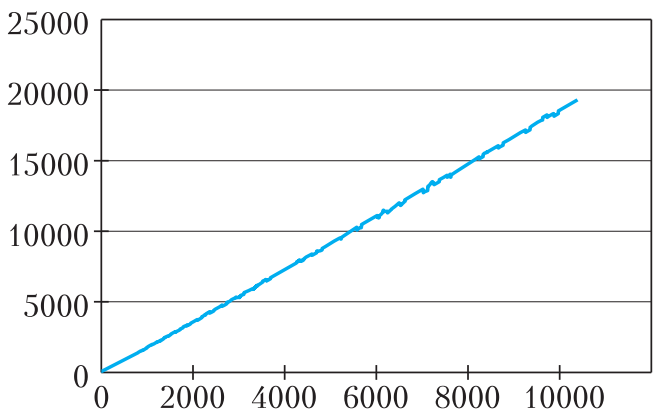

Рис. 3. Скользящее среднее разностей между последовательными точными степенями

«точки отсчета» $x$. Для последовательности точных квадратов, в силу равенства $(n+t)^{2}-n^{2}=2 n t+t^{2}, \quad$ скользящее среднее представляло бы собой линейную функцию. На рисунке видно, что более высокие степени вносят некоторые, впрочем не очень заметные, «биения».

А могут ли встретиться много подряд идущих натуральных чисел, каждое из которых - точная степень (в принципе, теорема 2 этого не запрещает)? Следующее упражнение дает отрицательный ответ на этот вопрос.

Упражнение 4. Докажите, что не существует четырех идущих подряд натуральных чисел, являющихся точными степенями.

Указание. Одно из двух последовательных четных чисел не делится на 4.

В 2002 году Преда Михайлеску доказал так называемую гипотезу Каталана о том, что 8 и 9 являются единственной парой соседних натуральных чисел, являющих- ся точными степенями (см., например, статью В.Сендерова и Б.Френкина «Гипотеза Каталана» в «Кванте» № 4 за 2007 г.). Более общий вопрос о том, что расстояние между соседними точными степенями стремится к бесконечности с возрастанием порядкового номера, остается открытой проблемой.

Упражнение 5. Покажите, что эта гипотеза эквивалентна следующему утверждению: для любого натурального $k$ существует лишь конечное множество решений уравнения $x^{m}-y^{n}=k$ в натуральных $x, y, m \geq 2, n \geq 2$.

Еще более общая гипотеза Пилаи гласит, что для любых натуральных $a, b, k$ существует лишь конечное множество решений уравнения $a x^{m}-b y^{n}=k$ в натуральных $x, y, m \geq 2, n \geq 2$.

\section{Прогресс с прогрессиями}

Как мы видели, длинных отрезков, т.е. арифметических прогрессий с разностью 1, состоящих из НЕстепеней, не существует. А как насчет (непостоянных) арифметических прогрессий с произвольной разностью? Классический результат (возможно, впервые доказанный Ферма) гласит, что не существует четырехчленной арифметической прогрессии из точных квадратов (об усилении этого результата см. также задачу М2025 «Задачника «Кванта» в №6 за 2006 г.). А для любого $n \geq 3$ не существует арифметической прогрессии из трех точных $n$-х степеней. Для $n=$ $=3$ и $n=4$ этот факт был доказан более 100 лет назад Кармайклом. Случай произвольного $n \geq 3$ был доказан сходными методами через несколько лет после доказательства большой теоремы Ферма.

Тем не менее, в натуральном ряду существуют сколь угодно длинные арифметические прогрессии из точных степеней. Построить такие прогрессии можно так. На начальном этапе конструирования возьмем произвольную прогрессию из $N$ натуральных чисел, например $a_{1}=1$, $a_{2}=2, \ldots, a_{N}=N$, и набор простых (или попарно взаимно простых) чисел $p_{1}, p_{2}, \ldots, p_{n}$. Идея - подобрать множитель $M$ вида $M=a_{1}^{s_{1}} \ldots a_{N}^{s_{N}}$ так, чтобы в про- 
грессии $M a_{1}, M a_{2}, \ldots, M a_{N}$ число на $i$-м месте являлось бы точной $p_{i}$-й степенью. Для этого достаточно подобрать показатели $s_{1}, \ldots, s_{N}$ так, чтобы $s_{j}$ делилось на $p_{i}$ при $j \neq i$ и $s_{i}+1$ делилось на $p_{i}$.

Упражнение 6. Реализуйте указанный план.

Указание. Воспользуйтесь китайской теоремой об остатках.

Для сравнения вспомним знаменитый результат 2004 года - теорему Грина-Тао о наличии в натуральном ряду сколь угодно длинных арифметических прогрессий из простых чисел. Следующее упражнение показывает, что в длинных арифметических прогрессиях из простых чисел или из точных степеней должны быть очень большие разности, несоизмеримо больше, чем количество членов прогрессии.

Упражнение 7. Пусть дана арифметическая прогрессия с разностью $d$, состоящая из $2 p$ натуральных чисел, где $p$ - простое число. Докажите, что в каждом из случаев а), б) $d$ делится на $p$.

a) Все члены прогрессии - простые числа.

б) Все члены прогрессии - точные степени.

(См. также задачу М2202 «Задачника «Кванта» в №6 за 2010 г.).

Указание. Если $d$ не делится на $p$, то в прогрессии длины $p$ встретятся все остатки по модулю $p$, значит, в прогрессии длины $2 p$ найдутся 2 числа, делящихся на $p$.

Теперь займемся бесконечными арифметическими прогрессиями $a+n d, n=0,1$, $2, \ldots$, где $a$ и $d-$ фиксированные натуральные числа, так что $d$ - разность прогрессии, а $a$ - ее первый член. Заметим, что все члены прогрессии с разностью $d$ дают один и тот же остаток при делении на $d$.

Знаменитая теорема Дирихле гласит, что при НОД $(a, d)=1$ в бесконечной арифметической прогрессии $a+n d$ присутствует бесконечное количество простых чисел. А что можно сказать о точных степенях и НЕстепенях в арифметической прогрессии?

Из теоремы 1 можно вывести, что в любой бесконечной арифметической прогрессии $a n+b$, где $a, b$ - данные положительные числа, существует бесконечное количество НЕстепеней. Несложно дать и конструктивное обоснование этого факта (см. упражнение ниже).

Упражнение 8. Пусть $p$ - простое число, на которое не делится $d$. Докажите, что в прогрессии $a+n d$ найдутся числа, делящиеся на $p$, но не делящиеся на $p^{2}$.

Указание. В такой прогрессии встретятся все остатки при делении на $p^{2}$.

Не так сложно привести примеры бесконечных арифметических прогрессий, в которых нет точных степеней.

Упражнение 9. Сделайте это.

Указание. Используйте идею упражнения 1. Разность прогрессии можно взять равной $p^{2}$.

Однако если первый член и разность прогрессии взаимно просты, то точные степени в прогрессии найдутся.

Теорема 3. Пусть $a, d-$ взаимно простыле натуральные числа. Тогда среди чисел вида $a+$ nd найдется точная степень.

Доказательство. Если $a=1$, в нашей прогрессии будет всякая степень вида $(1+n d)^{k}$.

Если же $a>1$, то в прогрессии можно отыскать степень числа $a$. Действительно, в ряду чисел $a, a^{2}, a^{3}, \ldots$ найдется пара чисел $a^{t}$ и $a^{s}, t<s$, дающих одинаковые остатки при делении на $d$. Тогда $a^{s}-a^{t}=a^{t-1}\left(a^{s-t+1}-a\right)$ делится на $d$. В силу взаимной простоты $a$ и $d$ получается, что $a^{s-t+1}-a$ делится на $d$, т.е. $a^{s-t+1}-a=d n$, или $a^{s-t+1}=a+d n$. Значит, $a^{s-t+1}$ и есть искомая степень.

\section{Упражнения}

10. Докажите, что бесконечная арифметическая прогрессия из натуральных чисел, содержащая по крайней мере одну точную $k$-ю степень, содержит и бесконечное количество точных $k$-х степеней.

(См. также, например, задачу М2059 «Задачника «Кванта» в №5 за 2007 г.)

Указание. Вместе с $c^{k}$ прогрессия должна содержать число $(c+d)^{k}$.

11. а) Докажите, что утверждение теоремы 3 перестает быть верным после замены слов «точная степень» на «точный квадрат». (Иными словами, существуют прогрессии $a+d n$, где 
НОД $(a, d)=1$, не содержащие точных квадратов.)

б) Докажите, что утверждение теоремы перестает быть верным после замены слов «точная степень» на «точная $k$-я степень» (для фиксированного $k \geq 2$ ).

Указание. Достаточно предъявить $d$ такое, что $k$-е степени пробегают не все возможные остатки при делении на $d$ (или, эквивалентно, среди чисел $0^{k}, 1^{k}, \ldots,(d-1)^{k}$ найдутся числа, дающие одинаковые остатки при делении на $d$ ).

Возвратимся еще раз к теореме Дирихле о простых числах в арифметических прогрессиях. На самом деле, эта теорема имеет красивое усиление, которое в виде гипотезы формулировали Лагранж и Дирихле, а доказал Валле-Пуссен. Неформально, это усиление гласит, что для любого натурального $d$ простые числа «распределены поровну» между остатками, взаимно простыми с $d$ (скажем, на большом начальном отрезке натурального ряда простых чисел вида $3 d+1$ и вида $3 d+2$ «примерно поровну»). Формальное утверждение таково. Для каждого $t \in\{1,2, \ldots, d-1\}$ такого, что НОД $(t, d)=1$, определяется функция $\pi_{d, t}(x)$, равная количеству простых чисел вида $t+n d$, не превосходящих $x$. Тогда $\lim _{x \rightarrow+\infty} \frac{\pi_{d, t}(x)}{\pi_{d, 1}(x)}=1$.

Заметим, что обобщить теорему 3 в таком же ключе не удается. Например, в прогрессии $3 d+1$ «гораздо больше» точных степеней, чем в прогрессии $3 d+2$, так как в последней нет точных квадратов.

\section{Удивительные суммы}

Завершая прогулку по королевству, покажем, как вычислить некоторые (бесконечные) суммы, где суммирование производится по всем НЕстепеням (или, наоборот, по точным степеням). Например, верна следующая теорема.

$$
\text { Теорема 4. } \sum_{m-\text { НЕстепень }} \frac{1}{m^{2}-1}=\frac{\pi^{2}}{6}-1 \text {. }
$$

Последовательность НЕстепеней весьма нерегулярна, поэтому возможность найти точное значение подобной суммы может по праву вызвать удивление. Но сейчас секрет будет раскрыт: используя идею «сте- пенного решета», мы сможем трансформировать различные суммы в суммы с «HEстепенным» индексом суммирования. Приведем соответствующую выкладку, опуская объяснения, почему в данном случае возможно изменение порядка слагаемых в данной бесконечной сумме (знакомые с анализом читатели могут дать строгое обоснование).

Как известно, $\sum_{n=1}^{\infty} \frac{1}{n^{2}}=\frac{\pi^{2}}{6}$. Отсюда

$$
\begin{aligned}
& \frac{\pi^{2}}{6}-1=\sum_{n=2}^{\infty} \frac{1}{n^{2}}= \\
& \quad=\sum_{m-\text { НЕстепень }}\left(\frac{1}{m^{2}}+\frac{1}{\left(m^{2}\right)^{2}}+\frac{1}{\left(m^{3}\right)^{2}}+\ldots\right) .
\end{aligned}
$$

Сумма геометрической прогрессии в скобках равна $\frac{1}{m^{2}-1}$, откуда и получаем нужный результат.

Конечно, тождества, аналогичные равенству из теоремы 4, можно вывести и для более общих сумм вида

$$
\sum_{m-\text { НЕстепень }} \frac{1}{m^{s}-1} .
$$

Упражнение 12. Вычислите

$$
\sum_{q>1 \text { - точная степень }} \frac{1}{q^{2}-1} .
$$

Указание. Используйте равенство

$$
\sum_{n=2}^{\infty} \frac{1}{n^{2}-1}=\frac{1}{2} \sum_{n=2}^{\infty}\left(\frac{1}{n-1}-\frac{1}{n+1}\right)=\frac{3}{8} .
$$

Предлагаем читателю проделать похожие преобразования с суммой $\sum_{n=1}^{N} \frac{1}{n}$ (вместо суммы $\sum \frac{1}{n^{2}}$ из теоремы 4) и вывести красивое равенство, известное как формула Гольдбаха или теорема Гольдбаха-Эйлера (Goldbach-Euler theorem):

$$
\sum_{m>1 \text { - точная степень }} \frac{1}{m-1}=1 .
$$

Автор благодарит редакцию за предложенные улучшения и дополнения в разделах об асимптотике и о прогрессиях, а также добавление упражнений. 